\title{
QUALITY OF SERVICE AND MOBILITY AWARE IN-VEHICLE TELESCREEN SERVICE ARCHITECTURE
}

\author{
Ghulam Sarwar, Farman Ullah, Hyun-Woo Lee, Won Ryu, Sungchang Lee
}

Original scientific paper This paper presents an architecture for providing quality of service (QoS) and mobility-aware telescreen services in vehicular networks, named "in-vehicle telescreen" (IVT). Providing seamless imagery effect is a key challenge in the distribution of high-resolution and delay-sensitive infotainment IVT services under mobile and varying network conditions. In the IVT architecture, new functions of information and service quality management are introduced to manage the user registrations, service advertisements, network information, and service quality. We propose an adaptive selection of application QoS parameters and an IVT handover decision mechanism for providing seamless imagery effect and enhance user's multimedia perception. The proposed architecture can provide a sustainable channel rate based selection of content source, media resolution, and codec to improve the perceived visual quality. Furthermore, the paper presents a Link Expiration Time and Received Signal Strength Indicator based IVT handover decision mechanism that can minimize the number of handovers by selecting a stable path. An extensive simulation is performed to show the effectiveness of the proposed mechanisms.

Keywords: handover decision mechanism; in-vehicle telescreen; quality-of-experience; quality-of-service; vehicular networks

\section{Arhitektura kvalitete usluge i usluge tele-ekrana u pokretnim voznim mrežama}

Izvorni znanstveni članak

U radu se predstavljena arhitektura za osiguranje kvalitete usluge (quality of service - QoS) i usluge tele-ekrana u voznim mrežama koje reagiraju na pokretljivost, nazvane teleekran unutar vozila - "in-vehicle telescreen" (IVT). Osiguranje nepromjenljive slike je ključni izazov u pružanju IVT usluga visoke rezolucije u mobilnim uvjetima i uvjetima variranja mreže. U IVT arhitekturi uvode se nove funkcije upravljanja kvalitetom informacija i usluga u vođenju registracije korisnika, oglašavanju usluga, obavijesti o mreži, i kvaliteti usluge. Predlažemo prilagodljiv izbor QoS parametara te mehanizam za donošenje odluke o prekapčanju IVT kako bi se osigurala čista slika i poboljšala multimedijska percepcija korisnika. Predloženom se arhitekturom može osigurati izbor izvora sadržaja zasnovan na prosječnoj kanalskoj brzini, rezolucija medija i kodek (koder-dekoder) za poboljšanje postojane vizualne kvalitete. Nadalje, u radu se predstavlja mehanizam za donošenje odluke o prekapčanju IVT temeljen na pokazivaču vremena isticanja veze i pokazivaču snage primljenog signala koji može smanjiti broj prekapčanja izborom stabilne staze. Provedena je opsežna simulacija kako bi se pokazala učinkovitost predloženih mehanizama.

Ključne riječi: mehanizam za donošenje odluke o prekapčanju; teleekran u vozilu; kvaliteta iskustva; kvaliteta usluge; vozne mreže

\section{Introduction}

Telescreen provides the remote playback of highresolution data to large-scale displays. Telescreen also termed as digital signage (DS) entered in daily life routines such as retail services, third-party advertisement, entertainment, wayfinding, education, and transportation, and its benefits in transportation will double in the next five years [1]. The DS market has grown to about 35 billion dollars around the world [2]. The global trend of migration from rural to urban areas has become more obvious nowadays. The metropolitan area people rely on the buses, cars and trains and spend most of their time in travelling. The rapid development of communication technologies enables to provide real-time infotainment services in-vehicle that open a new era of business models and opportunities. In-vehicle telescreen (IVT) can provide both safety and comfort services to travellers and drivers such as collision and lane change warning, traffic violation warning, parking alerts, news, multimedia and advertisement services. Safety services have high priority and require less bandwidth while the IVT infotainment services require high bandwidth and have strict QoS requirements. The main challenge in providing IVT infotainment services in urban areas is to provide seamless imagery effect in the varying network conditions. In this paper, we propose a QoS and mobilityaware IVT service architecture that bounds the end-to-end delay and minimizes the delay violation probability to improve the user' experience in a highly mobile and dynamic network conditions. We adaptively select the application QoS parameters such as source, codec, and resolution according to the network conditions to improve the service quality.

In vehicular networks, the wireless communication capability of vehicles enables to form a network with other vehicles and roadside infrastructure nodes while travelling on the road. The IVT On-Board Unit (OBU) displays the infotainment services and provides an interface to communicate with the infrastructure nodes (V2I, I2V) for providing internet connectivity and with other vehicles (V2V) to extend the coverage area. The existing cellular or WiFi networks have been employed to support vehicular communication [3, 4]. The cellular network provides high bandwidth and long-range communication at a higher cost while WiFi offers inexpensive bandwidth but requires enough roadside units (RSUs) for service continuity. The IEEE 802.11p/WAVE technologies and standards [5, 6] have been proposed to support the delay-sensitive and high-priority delivery requirements of safety services, and can support real-time infotainment services [7]. This paper focuses on the communication with the infrastructure nodes and adapts the WAVE short message protocol (WSMP) specified in the WAVE standard [6, 17] for managing and providing telescreen services in vehicular networks.

QoS refers to the system's capability to assign resources such as bandwidth, processing, and memory, according to user requirements. QoS is a measure of service availability and transmission quality of a network. Vehicular networks are characterized by high-speed mobility and heterogeneous network conditions that cause 
problems in achieving the QoS requirements of highresolution and delay-sensitive IVT multimedia services. The high-speed mobility of vehicles can increase the hop distance from the content source that may increase the packet delay and loss ratio. In this paper, we consider multiple telescreen content sources (TCS) and propose a dynamic source switching algorithm to minimize the network latency. Compression algorithms such as MPEG2, MPEG-4, H.264, and SVC, provide different levels of compression and are used to utilize the network resources. The varying topology and network conditions increase the packet drop ratio (PDR) and average packet delay (APD) which necessitate the adaptive selection of application QoS parameters to ensure the required visual quality to end users. Increasing the compression ratio lowers the bandwidth requirement, but a little distortion in a signal carrying highly compressed data results in lower visual quality. We propose QoS and mobility aware media resolution and codec selection schemes that consider the channel and traffic conditions to ensure better service quality.

Handover/Handoff management is the process of transferring a mobile user from one cell to another without service disruption. Handoff can be horizontal or vertical. Horizontal handoff refers to the mobility of a user between cells in the same mobile network while vertical handoff occurs between cells belonging to different networks. The handover decision is either based on the received signal strength indicator (RSSI) or link expiration time (LET) metric. RSSI is the measure of the power level from the radio access point and LET estimates the time to move out of the transmission coverage area. Taking handover decisions solely based on the RSSI from possibly multiple RSUs may cause unnecessary handovers as RSSI does not consider the direction of the moving nodes. Similarly, handover decision based on the LET metric may avoid a necessary handover in an urban area as it does not consider roadside obstacles that may have influence on the receiving signal strength. In this paper, we propose an efficient IVT handover decision mechanism based on both the RSSI and LET parameters to select a link with a longer expiration time and enough signal strength.

We organize the rest of the paper as follows: Section 2 presents the related works, and Section 3 presents the proposed IVT service architecture. We describe the handover decision mechanism, QoS parameters estimation and the algorithm for a sustainable rate based selection of application QoS parameters in Section 4. Section 5 demonstrates the simulation results and finally; we conclude the paper in Section 6 .

\section{Background and related works}

This section presents the background knowledge and related works on the digital signage (DS) architecture, vehicular networks, handover decision process, and QoS management of delay sensitive services in vehicular networks according to network conditions.

\subsection{Review of DS architecture and services}

Most of the existing telescreen/DS provides the remote controlled rendering and distribution of highresolution multimedia content to a group of displays that provides a single seamless imagery effect $[8,9]$. The declining prices of DS hardware, self-adaptability of content layout, and the shift towards greater interactivity are the main success factors of DS service. DS service providers merge the content from multiple sources such as advertisements, TV broadcasting, newspapers, and government announcements, and distribute them to trains, buses, transport terminals and hospitals instead of separate displays in public places for each [10]. Muller et al. [11] presented a DS with an installed camera to detect the user's presence and learn the preferences of the users to change the content accordingly. In previous works [9], we proposed the control framework for interactive DS that allows users to interact with the display using their multiple hand-held (N-Screen) devices. The system adapts the contents according to display layout and network conditions to improve the Quality-of-Service (QoS). This paper proposes a novel IVT architecture that extends the coverage area of telescreen to vehicular networks and improves the travel experience.

\subsection{Vehicular networks and handover decision}

Vehicular networks are usually short range communication networks, and the communicating devices can communicate directly with each other (V2V) [3] or with/through the infrastructure (V2I) [29] using the installed OBUs. Vehicular networks are characterized by the high-speed mobility, directions, and heterogeneous wireless network conditions that cause frequent route disruptions and changes in the network topology [12]. The high-speed mobility causes frequent handovers in vehicular networks. Most of the existing works on mobility management $[16,25]$ focus on layer- 3 schemes to extend the support of Mobile IP (MIP) to vehicular networks but not on the handover decision parameters. The handover decision is mostly based on either of the RSSI [14] or LET [30, 15] metric. To efficiently support the dissemination of high resolution and delay sensitive telescreen content in urban areas, we propose a handover decision mechanism based on both the RSSI and LET parameters to select a stable link and avoid unnecessary handovers.

\subsection{QoS management and delay-sensitive services in vehicular networks}

QoS management schemes mainly focus on the medium access control (MAC), network and application layer procedures to adapt the session flows according to the network conditions. The dynamic network conditions such as path loss, interference, shadowing effect and congestion cause issues in achieving the QoS requirements of multimedia services. Miclael-Oche et al. [13] proposed a MAC layer protocol that considers channel conditions to ensure the QoS requirements of real-time multimedia services. Amadeo et al. [7] proposed a MAC protocol to provide controlled-access functionalities to ensure the QoS requirements of service subscribers in IEEE 802.11p. The MAC layer procedures consider the wireless channel conditions to adapt the QoS but cannot accommodate the effect of wired network congestion on the QoS and user QoE. In [18], the authors 
proposed a network layer mechanism that adapts the QoS by the addition/deletion of media flows of a session upon terminal mobility. Many researchers $[19,20]$ focus on the adaptive selection of application QoS parameters according to network conditions during heterogeneous network mobility. These schemes may not be readily applicable in a vehicular environment due to the highspeed, predictable directions, and obstacles in urban and suburban areas. A cross-layer trigger-management framework was proposed in [19] to improve the QoS by providing network information to the resource management entity of the network. Y. C. Yee et al. [20] proposed an adaptive codec switching scheme to improve the network QoS during mobility across heterogeneous networks. The recently developed encoding schemes such as H.264 provide high efficiency in terms of visual quality and bandwidth consumption compared to MPEG-4 in good channel conditions. As the packet drop ratio, jitter and delay increases, MPEG-4 provides a higher visual quality than H.264 [32]. In this paper, we propose the dynamic selection of the appropriate content source and application QoS parameters to provide seamless service quality and improve user's QoE in a vehicular environment.

To differentiate from the previous researches, we can summarize the main contribution points of the paper as follows. (1) We propose a novel QoS \& mobility aware IVT service architecture for providing high-resolution and delay-sensitive telescreen services in vehicular networks.
(2) We propose network QoS and mobility-aware dynamic selection of telescreen content source and application QoS parameters that improve the perceived visual quality under dynamic network conditions. (3) To avoid unnecessary handoffs in urban and suburban areas, this paper proposes an efficient IVT handover decision scheme considering the RSSI and LET metrics for selecting a stable path. (4) We perform extensive simulations to show the feasibility and effectiveness of the proposed IVT system using the inet [21] and veins [22] frameworks of Omnet++ [23] (network simulator) and SUMO [24] (traffic simulator) with a realistic network environment and road traces.

\section{In-Vehicle Telescreen service architecture}

This section presents the proposed architecture to support the high-resolution and delay-sensitive telescreen services in vehicular networks. As mentioned previously, the main challenges for providing telescreen services in vehicular networks are to ensure better service quality in a highly mobile and dynamic network environment. Fig. 1 shows the conceptual architecture of IVT which extends our previous work [9] on DS to vehicular networks. To provide seamless imagery effect in IVT, we introduce the logical and functional nodes of the Telescreen Management System (TMS) and Quality-aware Service Manager (QSM).

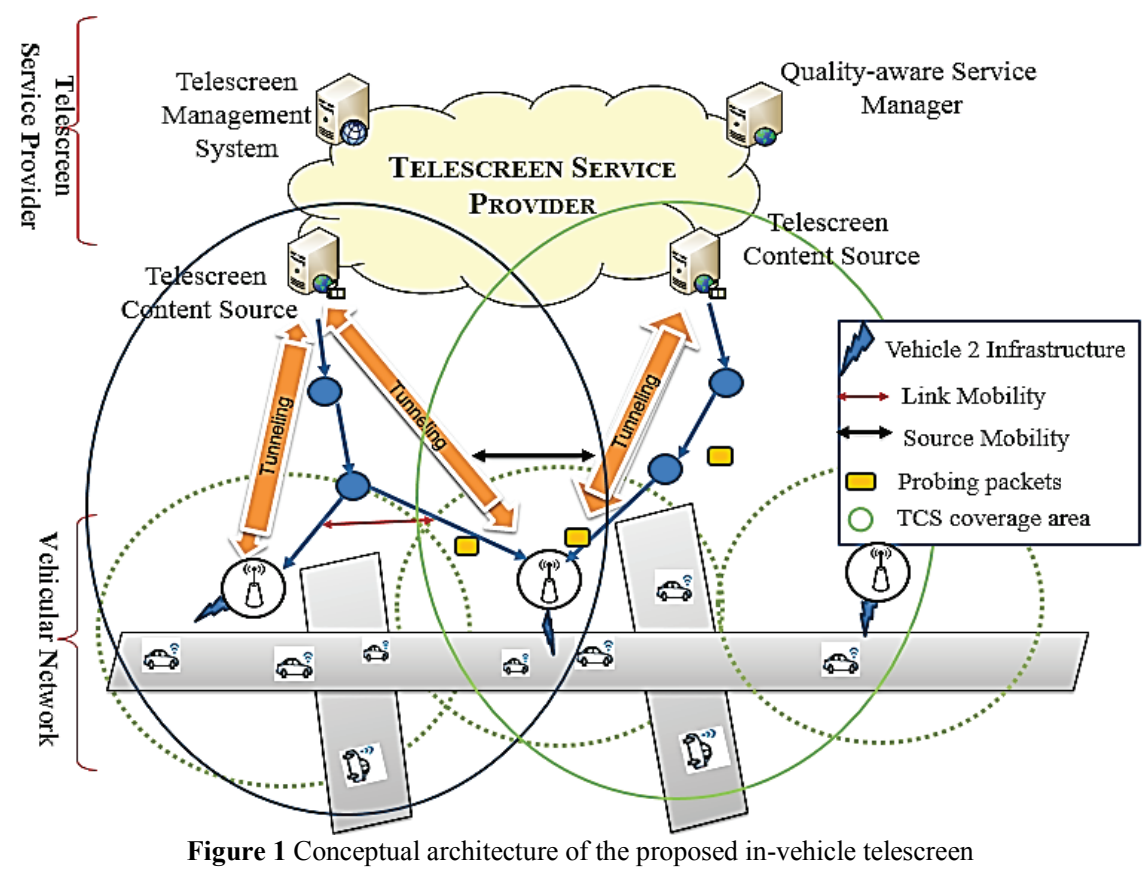

IVT can provide both commercial and noncommercial advertisements and infotainment services of different service providers. TMS is the central repository for user \& service registrations, advertisements, and mobility management. Based on service priority and location, TMS schedules service advertisements with the target RSUs. The WAVE service advertisement (WSA) includes ServiceInfo, ChannelInfo and WAVERouting Advertisement (WRA) segments that enable communication with the telescreen's infrastructure nodes.
The ServiceInfo segment consists of a content identifier, the IP address and port number of TMS. ChannelInfo contains the channel number, data rate, and transmission power while WRA includes the IP prefixes for address configuration. RSU periodically broadcasts WSA messages in its coverage areas for user's subscription. A vehicle can register to TMS by setting the 'ToDS' flag in the frame to let RSU forward the request to TMS. TMS registers the users and sends a registration response message to the vehicle with the IP addresses and port 
number of the QSM to apply for the content. User requests the desired content from the QSM with the desired source rate, delay limit, and delay violation limit. The QSM selects the appropriate TCS and content rate and requests the TCS to establish a session with the user. During mobility, when the signal strength falls below the minimum sensitivity level, the vehicle sends an association request to the best available RSU based on the RSSI and LET metrics. The RSU sends a binding-update message to TMS during the association process that may adapt the application QoS parameters according to the new channel conditions through QSM. QSM requests the content source to forward future streaming data to the node by tunnelling to the new RSU.

To enhance the network QoS and user's QoE, IVT maintains the end-to-end network QoS parameters such as $A P D$ and $P D R$ at the TCS. Maintaining accurate information about the network conditions (from a TCS to all destinations in the TCS's courage area) is cumbersome. Network QoS parameters can be estimated either by using the active or passive measurement strategy. The active strategy can accurately estimate the QoS parameters but generates extra traffic while passive strategy utilizes the existing traffic for the estimation. The additional traffic can increase the chances of collision and contention delay in the shared wireless channel. Thus, we separately estimate the QoS parameters of the wired and wireless networks. We use a packet train based probing technique for estimating the QoS parameters of wired networks and adjust the length of the packet train according to the network congestion. TCS periodically sends a probe train to the target RSUs in a coverage area, and RSUs report the reception timestamp of the last probe packet and the number of received probe packets of every train to the TCS. The content source estimates the $A P D_{\text {wired }}$ and $P D R_{\text {wired }}$ from the RSU's train-report and updates the network information at QSM. The network QoS parameters of the wireless channel of IVT service subscribers can be obtained from the periodic beacon messages. These beacon messages include the vehicle and wireless channel information such as the speed, direction, RSSI, and packet delay (PD). We estimate the $A P D_{\text {wired }}$ by averaging the PD of all received beacons in a time interval. Similarly, we approximate the PLR of the wireless network from the ratio of successfully received periodic beacons to the total number of expected beacons in the time interval. RSU reports the wireless channel conditions of IVT subscribers to the TCS nodes.

\section{Telescreen mobility and service quality management}

This section explains the feasibility of the proposed IVT system for providing the high-resolution infotainment services under highly mobile and varying network conditions. To provide seamless imagery effect and enhance user's QoE, IVT adaptively selects the application QoS parameters according to the dynamic network conditions. In this section, (1) we present the mechanisms for estimating the network QoS parameters. (2) We present the efficient handover decision mechanism based on the LET and RSSI parameters. (3) We explain the proposed algorithm for a sustainable rate based content source, media resolution, and codec selection to meet the delay requirements of IVT services.

\subsection{Service quality parameters estimation}

IVT extends infrastructure based DS services to vehicular networks. Providing seamless imagery effect in IVT demands an accurate model that characterizes the end-to-end traffic pattern and channel conditions of both wired and wireless networks. Quality degradation strongly depends on the network parameters such as bandwidth, packet loss and queuing delay and transmission delay. The end-to-end $P D R$ and $A P D$ can be expressed as in Eq. (1).

$$
\left\{\begin{array}{l}
P D R=P D R_{\text {wired }}+P D R_{\text {wireless }} \\
A P D=A P D_{\text {wired }}+A P D_{\text {wireless }}
\end{array}\right.
$$

To estimate $A P D_{\text {wired }}$ and $P D R_{\text {wired }}$, the TCSs periodically send probing packet trains to RSUs in their designated coverage areas. The $A P D_{\text {wired }}$ can be computed using Eq. (2).

$$
A P D_{\text {wired }}=\frac{t_{N}-t_{1}}{N}
$$

where $t_{1}$ denotes the starting time of the transmission of the first probe packet and $t_{N}$ denotes the reception time of the last probe packet of the train having $N$ number of packets. Let $M$ denote the total number of successfully received probe packets of the train at an RSU. The $P D R_{\text {wired }}$ can be computed using Eq. (3).

$$
P D R_{\text {wired }}=\frac{N-M}{N}
$$

IVT is a multihop network, and we consider the end-toend path consists of $n$ nodes. RSU estimates the expected link capacity and the queue size of the nodes followed by the probe packets and reports to the server. When a probe packet arrives at a node, the packet has to wait in queue if another packet is already in service. Let $\gamma$ represent the probability that the end-to-end delay is greater than zero can be estimated as the expected number of links at which the packet experienced the queuing delay as follows.

$$
\begin{aligned}
\gamma & =p r\{\text { end-to-end queuing delay }>0\} \\
& =\frac{1}{n} \sum_{i=1}^{n} \hat{q}_{i} \quad \hat{q}_{i} \in\{0,1\}
\end{aligned}
$$

where $\hat{q}_{i}$ is an indicator of the presence of queuing delay at link $i$. The expected link capacity of the end-to-end path can be estimated as the average of the capacities of links followed by the probe packets.

$$
E[\text { link capacity }]=\mathrm{E}[\mathrm{lc}]=\frac{1}{n} \sum_{i=1}^{n} r_{i}
$$


where $r_{i}$ is the transmission rate of node (link) $i$.

\subsection{Handover decision mechanisms}

The high-speed of vehicles and presence of obstacles in urban and suburban areas cause frequent handovers. Handover management is the process of seamlessly transferring a session from one link to another. LET and RSSI metrics have been widely used to define handover conditions. LET constitutes the stability of the link and considers the speed, direction, location, and transmission range of the vehicle and RSU. To avoid unnecessary handoffs and support high-resolution delay sensitive IVT services, we consider both the RSSI and LET parameters to select a stable link. The proposed mechanism selects the link having high signal strength and maximum expiration time. Nodes in the vehicular network can determine the RSSI from the periodic beacon messages. We assume that nodes have global positioning system (GPS). Let $\left(x_{i}, y_{i}\right)$ and $\left(x_{r}, y_{r}\right)$ denote the Cartesian coordinates of a vehicle $i$ and RSU $r$. Knowing the speed $v$ and direction $\theta$ of vehicle $i$ [28], and the maximum transmission range $R$ of the RSU, we can estimate the $L E T_{i, r}$ as in Eq. (6) [30].

$$
L E T_{i, r}=\frac{\left(\sqrt{\left(a^{2}+c^{2}\right) R^{2}-(a d-b c)^{2}}\right)-a b-c d}{a^{2}+c^{2}}
$$

where:

$$
\left\{\begin{array}{l}
a=v^{*} \cos (\theta) \\
b=x_{i}-x_{r} \\
c=v^{*} \sin (\theta) \\
d=y_{i}-y_{r}
\end{array}\right.
$$

\subsection{Algorithm for sustainable rate based source \& codec switching}

IVT multimedia services are delay-sensitive and have stringent QoS requirements. A packet exceeding its maximum delay limit is discarded. Recalling from [26], the probability that the APD at time $t$ will exceed the maximum delay limit, decreases exponentially with the increase in the delay limit. The arriving process at time instant $t$ served by the channel service could be guaranteed within a limited violation probability $\varepsilon$.The probability that the $A P D$ exceeds the maximum delay limit $D_{l}$ can be expressed as follows:

$$
\operatorname{pr}\left\{A P D \geq D_{l}\right\} \leq \varepsilon=\gamma^{*} \exp ^{-b(\mu)^{*} D_{l}}
$$

An increase in $\gamma$ would increase the delay violation probability and increase in the QoS component $b(\mu)$ would decrease the probability of delay limit violation. The parameter $b(\mu)$ is the decay rate and depends on the network transmission rates and queuing delays. The parameters $b(\mu)$ and $\gamma$ characterize the end-to-end delay experienced by a packet as follows [33]:

$$
\begin{aligned}
& \frac{\gamma}{b(\mu)}=E\left[D_{t}\right] \\
& b(\mu)=\frac{\gamma}{E\left[D_{t}\right]}=\frac{\gamma}{\gamma T_{r}+T_{m} Q}=\frac{\gamma \mu}{\gamma \mu T_{r}+Q}
\end{aligned}
$$

where:

$$
\begin{aligned}
& \mu=\text { source content rate } \\
& Q=\text { End-to-End Queue length }=\sum_{i=1}^{n} q_{i}
\end{aligned}
$$

$\mathrm{q}_{\mathrm{i}}$ is the queue length at node ' $\mathrm{i}$ '

$\operatorname{Tr}=$ Expected Tramission delay $=\frac{1}{E[l c]}$

The original capacity theorem (Eq. (7)) was proposed for single node model while the IVT is a multihop network. We approximated a single node model as a representation of the end-to-end path with end-to-end queue length and expected path's link capacity as in [34]. Our proposed scheme adjusts the source rate to minimize the delay bound violation as shown in Fig. 2. An increase in source rate $\mu$ would increase the queuing delay that increases the delay violation probability.

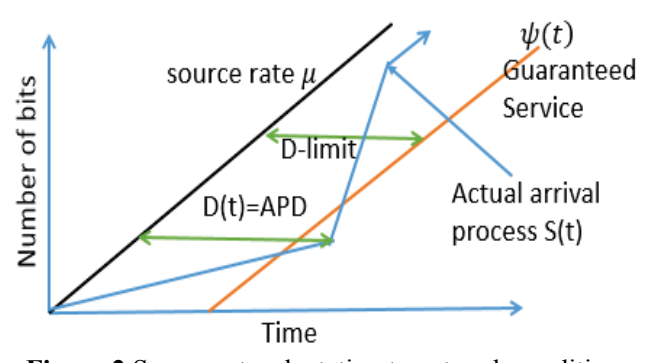

Figure 2 Source rate adaptation to network conditions

Fig. 3 shows the flowchart of the proposed algorithm for a sustainable rate based selection of appropriate source rate, codec, and media resolution to provide seamless imagery effect and to maintain user's QoE. The QSM selects the appropriate source that offers minimum delay violation probability. The selected source selects the appropriate codec and resolution that can ensure the required visual quality requirements and the channel can sustain in the current network conditions. We select the codec that can provide higher visual quality on user's device. We adapt the concept of ITU-T G.1070 [35, 36] to estimate the multimedia quality (Mean Opinion Score) on user's N-Screen device.

$$
M O S=1+\left(v_{3}-\frac{v_{3}}{\left[1+\frac{\mu}{v_{4}}\right] * v_{5}}\right)
$$


where $M O S$ is the perceived visual quality on user's device and is in the range of (1-5). We used the user's requested rate $\mu$ in the MOS estimation. The variables $v_{3}$, $v_{4}$, and $v_{5}$ are the model parameters that depend on the media codec, frame rate and display screen size. The algorithm selects user's preferred resolution level of the codec if the end-to-end delay is within the delay bound and visual quality requirement is satisfied. If the network conditions cannot ensure the visual quality requirements of the service for the requested rate, we adapt the codec and resolution as follows:

- We switch to another codec that can bound the endto-end delay and ensures that the MOS requirement is satisfied.

- Otherwise, we select a lower resolution, and select the codec that can provide better visual quality.

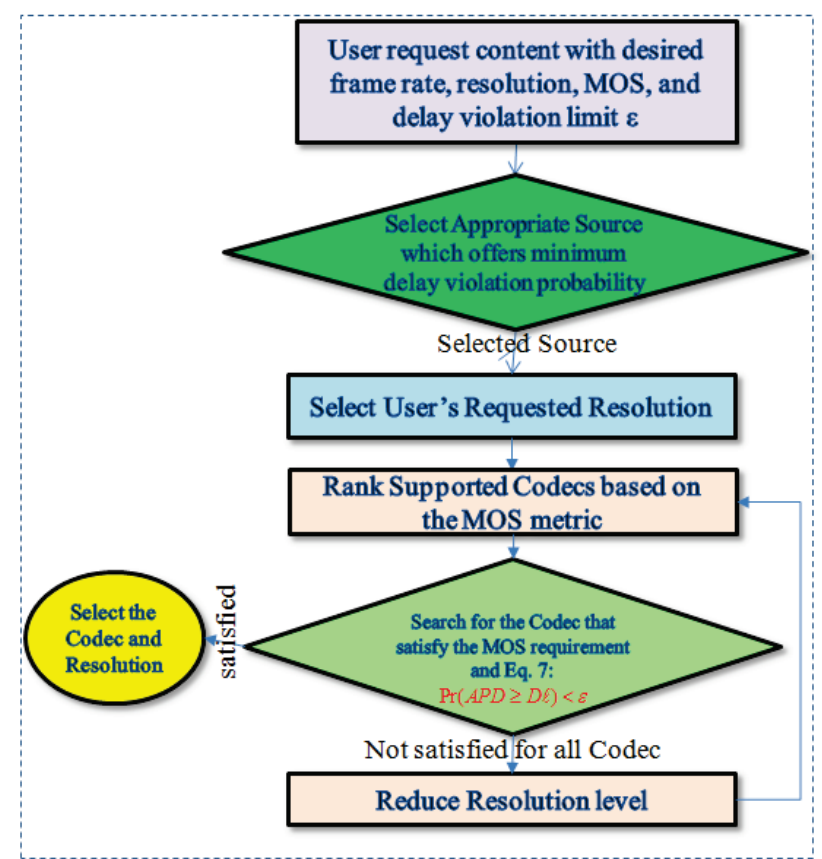

Figure 3 Flowchart of the adaptive parameters selection mechanism

\section{Simulation results}

To determine the effectiveness of the proposed QoS and mobility aware IVT system, this section demonstrates the extensive simulation results obtained by using the inet [21] and veins [22] frameworks of the Omnet++ [23] network simulator, and the SUMO [24] traffic simulator. Omnetpp implements the communication protocols of IVT, and the SUMO traffic simulator gathers information about vehicular traffic conditions such as the density, speed, direction, and road ID of vehicles from real road traces. The TraCI module of the veins framework bridges the Omnetpp and SUMO simulators to work together. We use MPEG-4 and H.264 encoded video traces of the same content in different resolutions to show the effect of the adaptive selection of application QoS parameters according to the varying network conditions on the perceived visual quality. Tab. 1 lists the simulation setup parameters. Fig. 4 shows the simulation environment with roadside obstacles.
Table 1 Symbols and Description

\begin{tabular}{|l|c|}
\hline \multicolumn{1}{|c|}{ Symbol } & Description \\
\hline MAC & IEEE $802.11 \mathrm{p}$ \\
\hline Frequency Band & $5,9 \mathrm{GHz}$ \\
\hline RSU Transmission Range & $300 \mathrm{~m}$ \\
\hline Beacon Frequency & $0,1 \mathrm{~ms}$ \\
\hline WSA Frequency & $1 \mathrm{~s}$ \\
\hline Beacon/WSA packet Size & 500 Bytes \\
\hline Transport Protocol & UDP \\
\hline Frame Rate & $30 \mathrm{fps}$ \\
\hline Probe Packet Size & $1000 \mathrm{Bytes}$ \\
\hline Probe Packets in Train & $2 \div 5$ \\
\hline Required MOS & 2,3 \\
\hline Delay Violation Probability & 0,001 \\
\hline$D_{\ell}$ & $0,033 \mathrm{~s}$ \\
\hline Max. Transmission Unit & $1500 \mathrm{Bytes}$ \\
\hline Bottleneck link Capacity & $5 \mathrm{Mpbs}$ \\
\hline Vehicle Density & $10 \div 20$ \\
\hline Vehicle Speed & $5 \div 50 \mathrm{mps}$ \\
\hline
\end{tabular}

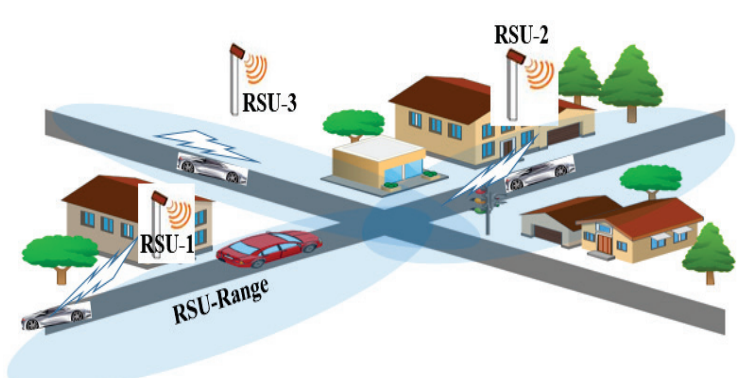

Figure 4 Simulation environment

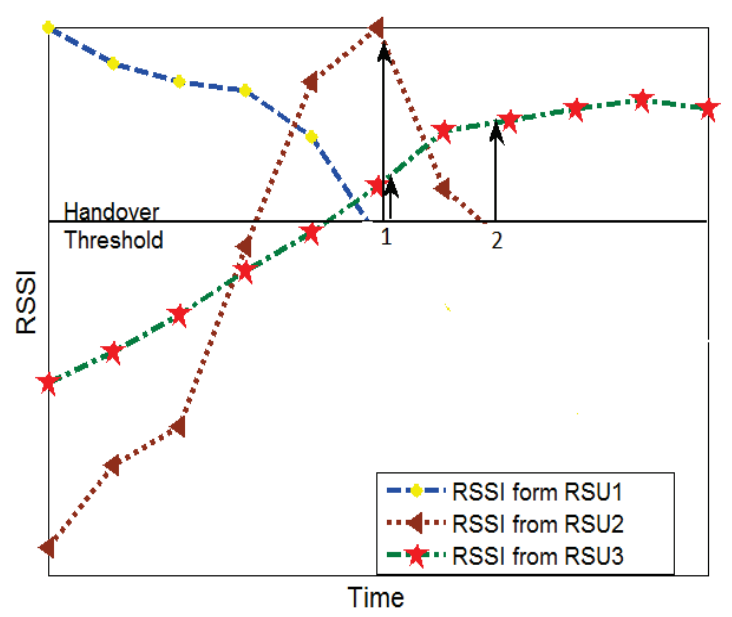

Figure 5 Proposed handover mechanism

Fig. 5 shows the effectiveness of the proposed handover decision scheme by plotting the RSSI from three RSUs while moving along the roadway. Initially, the vehicle is connected to the RSU1. When the receiving signal degrades, and RSSI reaches the threshold, the vehicle performs a handover to another available RSU. If the handover decision is solely based on the RSSI threshold, the vehicle needs to perform two handovers at points 1 and 2 to RSU2 and RSU3 respectively. Our proposed scheme considers both the LET and RSSI metrics and performs a handover to RSU3 at point 1 .

The proposed schemes select the appropriate content source and adapt the application QoS parameters according to varying network conditions to ensure the visual quality and delay requirements of IVT services. For 
comparing the effect of the adaptive selection of source, codec and media resolution on the user's QoE, we use peak signal to noise ratio (PSNR). PSNR compares the signal energy to the noise energy between the original sent and received frames $\left(F_{s}, F_{r}\right)$. PSNR can be expressed as[31]:

$$
\operatorname{PSNR}\left(F_{s}, F_{r}\right)=20 \cdot \log 10 \cdot \frac{V_{\text {max }}}{M S E\left(F_{s}, F_{r}\right)}, \mathrm{dB}
$$

where $V_{\max }=2^{p}-1$, and $p$ is the pixel colour depth. The increasing distance between the high-speed vehicles and the RSU and the presence of obstacles increase the PDR that reduces the PSNR. The visual quality of real-time multimedia services with PSNR less than $25 \mathrm{~dB}$ is considered to be poor and not acceptable. As depicted in Fig. 6 , the source selection criteria based on the minimum delay violation probability ensures better perceived visual quality. We select the codec that offers better visual quality and ensure that the MOS and end-to-end delay requirements are met.

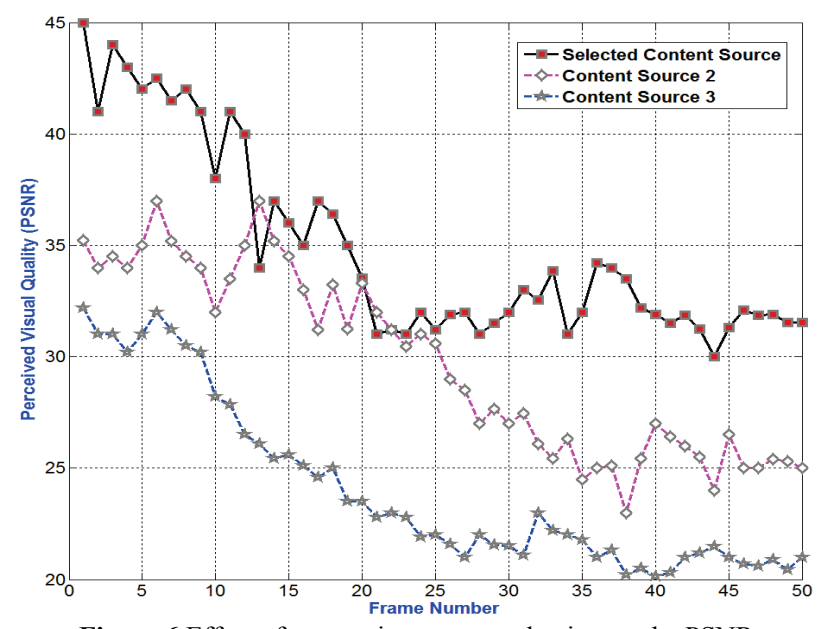

Figure 6 Effect of appropriate source selection on the PSNR

Fig. 7 compares the performance of different codecs in varying network conditions. In the experiment, we compared the H.264 and MPEG-4 codecs on the same device using the ITU-T G1070 model.

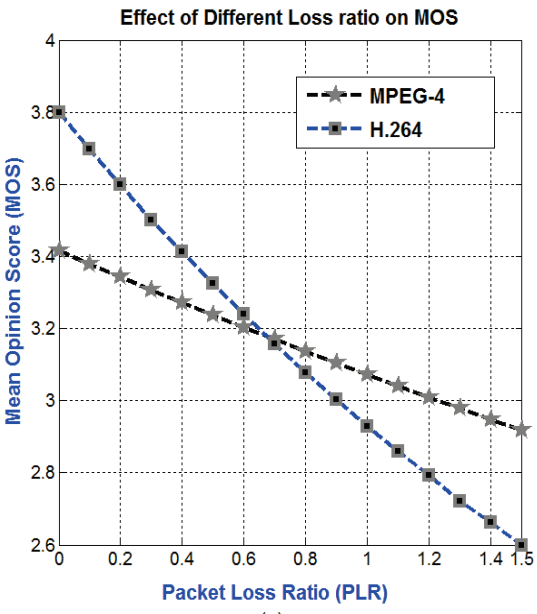

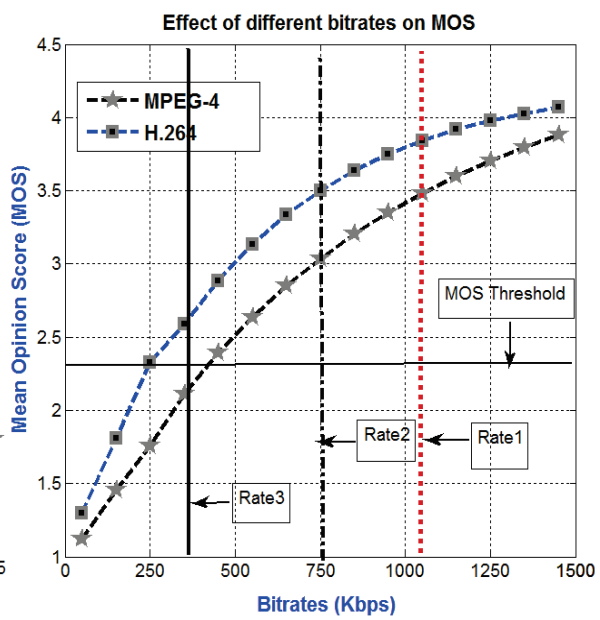

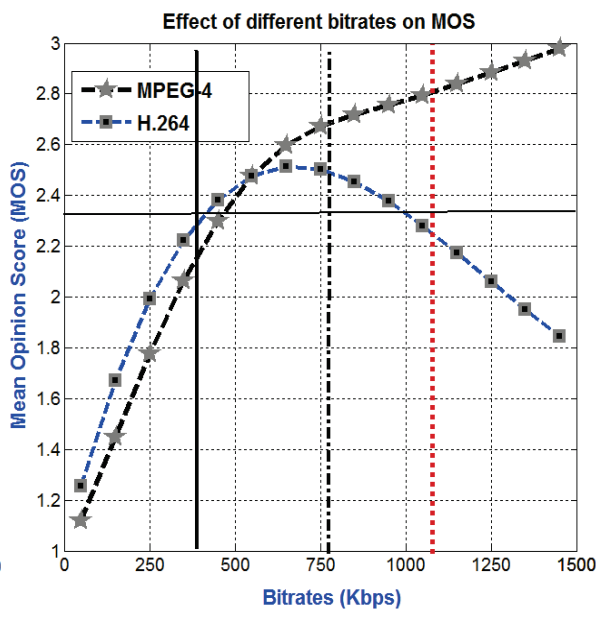

Figure 7(a) Codec performance at varying PLR: same device $=9,2$ inch, same bit rate $=1 \mathrm{Mbps}$, (b) codec performance at varying bit rate on: same device $=9,2$ inch, same PLR $=0 \%$,(c) codec performance at varying bit rate in the presence of PLR $=2 \%$, using ITU-T G. 1070 Model

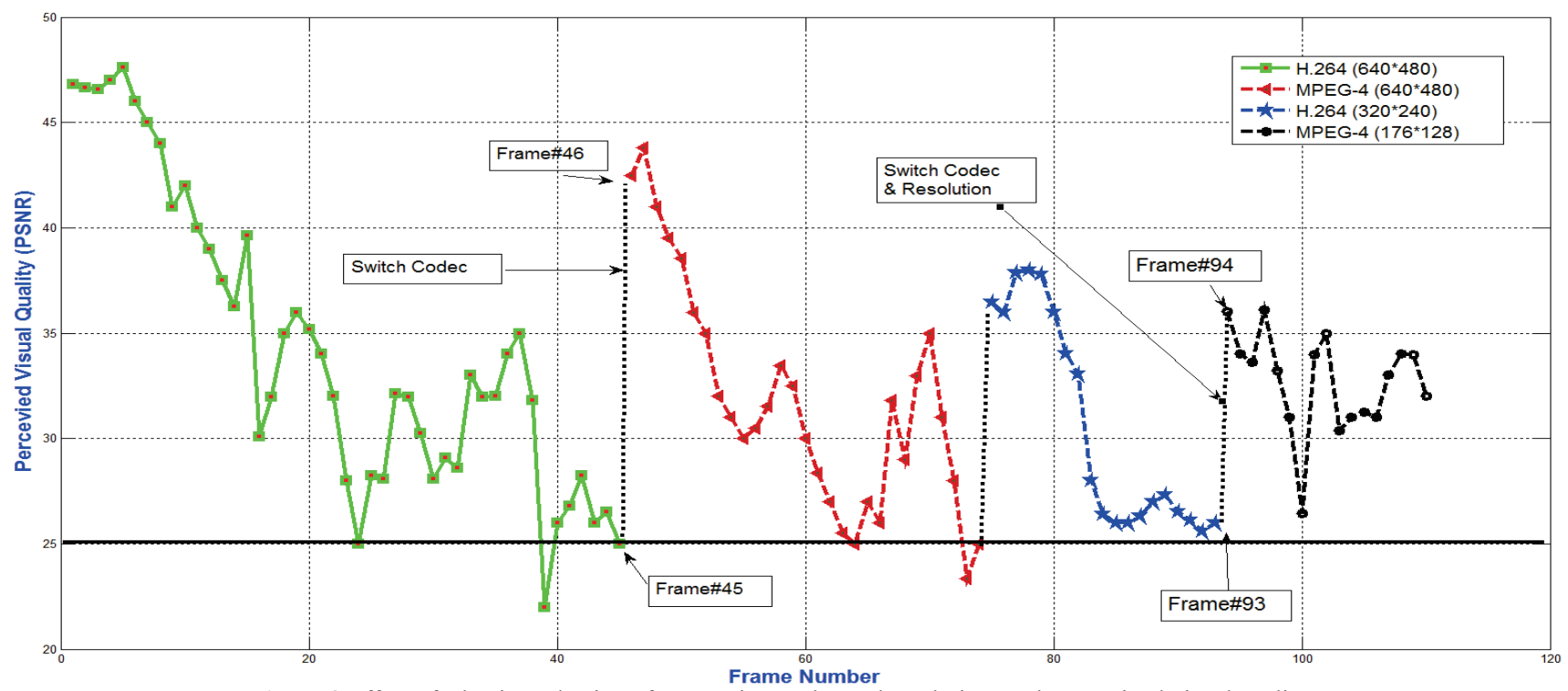

Figure 8 Effect of adaptive selection of appropriate codec and resolution on the perceived visual quality 
In Fig. 7a, we observed that at higher PLR increases, MPEG-4 can provide better visual quality than H.264. Fig. $7 b$ shows that in good channel conditions near RSU, H.264 offers better visual quality and support highresolution (Rate 1) stream. With the increase in PLR, the estimated perceived visual on the user's device falls below the threshold value. The proposed scheme switches to another codec that can ensure the required visual quality to the user subject to the sustainable channel rate. The system adaptively selects MPEG-4 that can ensure both the requested resolution and visual quality to users as shown in Fig. 7c. We use the Foreman video sequence in H.264 and MPEG-4 formats from [27] in different resolutions and bit rates. The proposed algorithm selects a high visual quality sequence under good channel conditions. The PDR and APD increase with the increase in the distance from the RSU and content source, which results in a lower visual quality. The smaller value of the QoS exponent $(b(\mu))$ increases the delay violation probability. Our proposed scheme adaptively switches the content codec and resolution to maintain the perceived visual quality. As depicted in Fig. 8, if the channel conditions cannot ensure the user's QoS requirements, the adaptive selection of appropriate codec and media resolution can provide better visual quality in the same channel conditions.

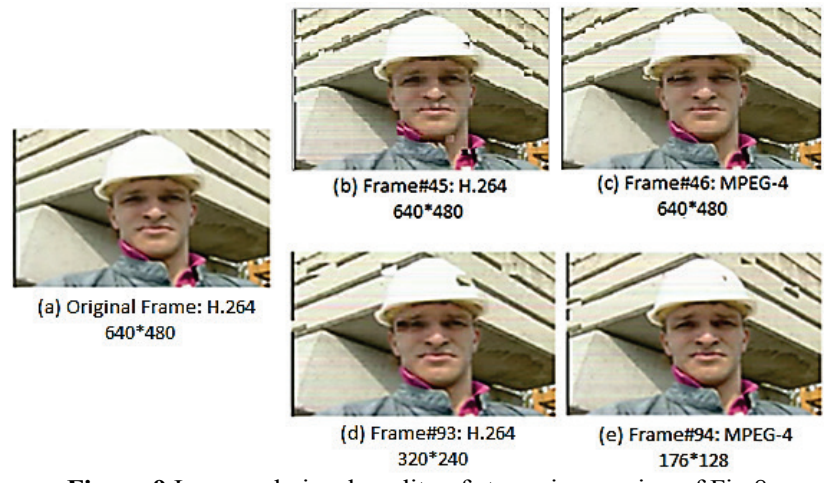

Figure 9 Improved visual quality of streaming session of Fig. 8

Fig. 9 visually demonstrates the effect of the proposed adaptive media resolution and codec selection scheme on the perceived visual quality of the streaming session of Fig. 8. Fig. 9a shows the original foreman video frame in H.264 format and $640 \times 480$ resolution. As depicted in Fig. $7 \mathrm{~b}$ and Fig. 7c, the adaptive selection of codecs according to network conditions can improve the visual experience of users. If none of the supported codecs can ensure the visual quality requirements of users of the current resolution level, our proposed scheme switches to a switch to a low-resolution level as shown in Fig. 7d and Fig. 7e.

\section{Conclusion}

In this paper, we proposed architecture for mobility and QoS-aware telescreen services in vehicular networks. The proposed system adapts the application QoS parameters to maintain the user's QoE in dynamic network conditions. We introduced the schemes for the estimation of network QoS parameters, and a logical node in the IVT architecture for managing the network information and selecting appropriate QoS parameters. The sustainable channel rate based media resolution, codec format, and source switching schemes minimized the delay violation probability of real-time IVT multimedia services in the dynamic network conditions. We considered both the RSSI and LET metrics for the selection of a stable link during link handover. Through simulations, we proved the necessity and effectiveness of the appropriate selection of content source, media codec, and resolution for providing seamless imagery effect under dynamic network conditions. This paper presented the I2V and V2I communication and considered IEEE $802.11 \mathrm{p} / \mathrm{WAVE}$ to provide IVT services. In future work, we are focusing on extending the architecture to multihop and heterogeneous network environments.

\section{References}

[1] The State of the Digital Signage Industry Survey. // Published by NetWorld Alliance, 2009.

[2] Digital Signage Market: Key Research Findings 2010.// Yano Research Institute Ltd.

[3] Santa, J.; Gomaz-Skarmeta, A. F.; Sanchez-Artigas, M. Architecture and evaluation of a unified V2V and V2I communication system based on cellular networks. // Computer Communications. (2008), pp. 2850-2861. DOI: 10.1016/j.comcom.2007.12.008

[4] Eriksson, J.; Balakrishnan, H.; Maddan, S. Cabernet: Vehicular Content Delivery Using WiFi. // Proc. ACM MobiCom, (2008), pp. 199-210. DOI: 10.1145/1409944.1409968

[5] Wireless LAN Medium Access Control (MAC) and Physical Layer (PHY) specifications Amendment: Wireless Access in Vehicular Environments. // IEEE Draft Std P802.11p, 2010.

[6] 1609.3-2010 IEEE Standard for Wireless Access in Vehicular Environments (WAVE) - Networking Services. // IEEE Std 1609.3-2010.

[7] Amadeo, M.; Campolo, C.; Molinaro, A. Enhancing IEEE $802.11 \mathrm{p} /$ WAVE to provide infotainment applications in VANETs. // Ad Hoc Networks, 2010.

[8] Renambot, L.; Rao, A.; Singh, R.; Jeong, B.; Krishnaprasad, N.; Vishwanath, V.; Chandrasekhar, V.; Schwarz, N.; Spale, A.; Zhang, C.; Goldman, G.; Leigh, J.; Johnson, A. SAGE: the Scalable Adaptive Graphics Environment. // Proceedings of WACE 2004.

[9] Ullah, F.; Sarwar, G.; Lee, H.; Ryu, W. Lee, S. Control Framework and Service Scenarios of Provisioning NScreen Services in Interactive Digital Signage. // Technical Gazette 21, 6(2014), pp. 1321-1328.

[10] Inoue, H.; Suzuki, K.; Sakata, K.; Maeda, K. Development of a Digital Signage System for Automatic Collection and Distribution of its Content from the Existing Digital Contents and its Field Trials. // Proceedings of IEEE International Symposium on Applications and the Internet, 2011. DOI: 10.1109/saint.2011.86

[11] Muller, J.; Exeler, J.; Buzeck, M.; Kruger, A.ReflectiveSigns: Digital Signs that Adapt to Audience Attention. // Proceedings of Pervasive 2009, Nara, 2009.

[12] Jarupan, B.; Ekici, E. A Survey of cross-layer design for VANETs. // Ad Hoc Networks, 2011. DOI: 10.1016/j.adhoc.2010.11.007

[13] Oche, M.; Noor, R. M.; Aghinya, J. I. Network centric QoS performance of IPTV transmission quality over VANETs. // Computer Communications, 2014.

[14] Marwart, S-N-K.; Meyer, S.; Weerwardane, T.; Goery, C. Congestion-Aware Handover in LTE Systems for Load 
Balancing in Transport Network.// ETRI Journal. 36, 5(2014), pp. 761-771. DOI: 10.4218/etrij.14.0113.1034

[15] Asefi, M.; Cespedes, S.; Shen X.; Mark, J-W. A Seamless Quality-Driven Multihop Data Delivery Scheme for Video Streaming in Urban VANET Scenarios. // IEEE ICC, 2011.

[16] Naor, Z. Fast and reliable handoff for vehicular networks. // Ad Hoc Networks. (2013), pp. 2136-2145. DOl: 10.1016/j.adhoc.2012.05.002

[17] Campoloand, C.; Molinaro, A. Improving V2R connectivety to provide ITS applications in IEEE $802.11 \mathrm{p} /$ WAVE VANETs. // Proceedings of 18th International Conference on Telecommunications, 2011.

[18] Cergueira, E.; Veloso, L.; Neto, A.; Curado, M.; Monteiro, E.; Mendes, P. Mobility management for multi-user sessions in next generation wireless systems.// Comput. Commun. 31, (2008), pp. 915-934. DOl: 10.1016/j.comcom.2007.12.019

[19] Makela, J. Towards seamless mobility with cross layer triggering. // Proceedings of the 18th Int. Syposium, PIMRC, pp. 1-5 2007. DOI: 10.1109/pimrc.2007.4394848

[20] Yee, Y. C.; Choong, K. N.; Low, A. L. Y.; Tan, S. W. SIPbased proactive and adaptive mobility management framework for heterogeneous networks.// J. Netw. Comput. Appl. 31, (2008), pp. 771-792. DOl: 10.1016/j.jnca.2007.09.002

[21] http://inet.omnetpp.org

[22] Sommer, C.; German, R.; Dressler, F. Bidirectionally Couped Network and Road Traffic Simulation for improved IVC Analysis. // IEEE Transactions on Mobile Computing. 10, 1(2011), pp. 2-15. DOI: 10.1109/TMC.2010.133

[23] Omnet++ simulator framework, <http://omnetpp.org/>

[24] I. of Transportation Systems at the German Aerospace Center, Simulation of urban mobility, $<\mathrm{http}$ ://sumo.sourceforge.net/>

[25] Cespedes, S.; Shen, X. IP Mobility Management for Vehicular Communication Networks: Challenges and Solutions.// IEEE Communications Magazine, 2011. DOI: 10.1109/MCOM.2011.5762817

[26] Wu, D.; Negi, R. Effective capacity: a wireless link model for support of quality of service.// IEEE transaction on Wireless Commun. 2, (2003), pp. 630-643.

[27] ftp://ftp.pixeltools.com/H264Examples/

[28] http://www.movable-type.co.uk/scripts/latlong .html

[29] Momeni, S.; Lai, J.; Wolfinger, B-E. Availability Evaluation of IPTV Services in Roadside Backbone Networks with Vehicle-to-Infrastructure Communication. // IEEE Conf., 2013. DOI: 10.1109/iwcmc.2013.6583817

[30] Benslimance, A.; Taleb, T.; Sivaraj, R. Dynamic Clustering-Based Adaptive Mobile Gateway Management in Integrated VANET-3G Heterogeneous Wireless Networks. // IEEE Journal of Selected Areas in Communications. 29, 3(2011), pp. 559-570. DOl: 10.1109/JSAC.2011.110306

[31] Ribeiro, V.; Riedi, R.; Baraniuk, R.; Navratil, J.; Cottrell, L. PathChirp: efficient available bandwidth estimation for network paths. // Proceedings. of Passive Active Measurements Workshop. 82003), pp. 1-11.

[32] Canovas, A.; Garcia, M.; Lloret, J.; Atenas, M.; Rizo, R. Improving IPTV QoE taking the suitable MPEG-2/MPEG4 Quantizer based on Jitter, Delay and lost packets measurements. // International Journal on Advances in Telecommunications. 3, 3-4(2010), pp. 129-139.

[33] Eckberg, A. E. Approximations for bursty and smoothed arrivals delays based on generalized peakedness. // Proceedings $11^{\text {th }}$ Int. Teletraffic Congress, Kyoto, Japan, Sept, 1985

[34] Kim, H. S.; Shroff, N. B. The notion of End-to-End Capacity and its Application to the estimation of End-toEnd Networks Delays. // Computer Network, 2005. DOl: 10.1016/j.comnet.2004.11.015
[35] ITU-T G.1070. Opinion model for video-telephony applications, 2007.

[36] Video Quality Analysis Software: www.e-model.org/EModelV2006+MM-ModelV2007.xls.

\section{Authors' addresses}

Ghulam Sarwar, PhD Student

Department of Information and Telecommunication Engineering, Korea Aerospace University,

Deogyang-gu, Goyang-si, Gyeonggi-do, 412-791, South Korea

E-mail: sarwar@kau.kr

\section{Farman Ullah, PhD}

${ }^{1}$ Department of Information and Telecommunication Engineering, Korea Aerospace University,

Deogyang-gu, Goyang-si, Gyeonggi-do, 412-791, South Korea

${ }^{2}$ Department of Electrical Engineering,

COMSATS Institute of Information Technology, Attock, Pakistan

E-mail: farmankttk@ciit-attock.edu.pk

Hyunwoo Lee, PhD, Research Engineer and Team Leader

Department of Smart Convergence, Electronics \&

Telecommunications Research Institute (ETRI),

Daejeon, South Korea

E-mail: hwlee@etri.re.kr

Won Ryu, PhD, Managing Director

Department of Smart Convergence, Electronics \&

Telecommunications Research Institute (ETRI)

Daejeon, South Korea

E-mail:wlyu@etri.re.kr

\section{Sungchang Lee, PhD, Professor}

(Corresponding author)

School of Electronics and Information Engineering,

Korea Aerospace University,

Deogyang-gu, Goyang-si, Gyeonggi-do, 412-791, South Korea

E-mail: sclee@kau.ac.kr 